\title{
Biochemie und Pathobiochemie des Kallikrein-Kinin-Systems der Organe
}

Dittmann, B., Geiger, R., Fink, E. (Abt. für Klin. Chemie und Klin. Biochemie, Chirurg. Klinik der Univ. München), Mann, K. (Med. Klinik II, Klinikum Großhadern, München), Fritz, H. (Abt. für Klin. Chemie und Klin. Biochemie, Chirurg. Klinik der Univ. München)

\section{Referat}

Das Kallikrein-Kinin-System ist ein im Organismus ubiquitär vorkommendes System (Übersichtsliteratur: Pisano u. Austen, 1976; Erdös, 1976; Sicuteri et al., 1976). Grundsätzlich ist zu unterscheiden zwischen dem Kallikrein-Kinin-System des Plasmas und dem der Organe. Beiden Systemen ist ein Zusammenspiel bestimmter Komponenten gemeinsam. Aus dem Substrat Kininogen wird durch Kallikrein - einer Kininogenase - Kinin herausgespalten (Bradykinin = Arg-Pro-Pro-Gly-Phe-Ser-Pro-Phe-Arg bzw. Kallidin = Lys-Bradykinin). Die Kinine stellen die eigentlichen Effektoren dar. Kininasen, das sind Kinin-abbauende Proteasen, und Inhibitoren der Kallikreine und Kininasen sorgen für die örtliche und zeitliche Regulierung des Kininspiegels (s. Abb. 1).

Die Kinine spielen eine wesentliche Rolle bei der Erregung glattmuskulärer Organe, wie Darm und Uterus. Sie führen zur Dilatation peripherer Gefäße und somit zur Blutdrucksenkung, sie erhöhen die Kapillarpermeabilität, erzeugen Schmerz und wirken chemotaktisch. Sie sind für die bei einer Entzündung auftretenden Erscheinungen wie dolor, calor, rubor mit verantwortlich und werden deshalb als Entzündungsmediatoren bezeichnet.

Der wesentliche Unterschied der beiden Systeme ist biochemisch-funktionell bedingt. Es gibt zwei kininogene: Das hochmolekulare oder HMW- und das niedermolekulare oder LMW-Kininogen. HMW-Kininogen ist das Substrat sowohl für Plasmakallikrein als auch für Organkallikreine, während LMW-Kininogen (entspricht $80 \%$ des gesamten Kininogens im Plasma) das bevorzugte Substrat der Organkallikreine darstellt. Das Organkallikrein-Kinin-System dürfte ganz generell als ein Transportmediator- und Transportinitiatorsystem an Membranen zu betrachten sein, das zudem vegetativ/hormonell gesteuert wird. Das HMW-Kininogen-Plasmakallikrein-Kinin-System hat eine zusätzliche bedeutende Funktion in der Blutgerinnung und Fibrinolyse. HMW-Kininogen und Plasmakallikrein sind nach unserem heutigen Wissen in erster Linie als Gerinnungsfaktoren zu betrachten (Kato et al., 1977).

Beide Kininogene sind einkettige saure Glykoproteine, deren $\mathrm{H}$ - und L-Ketten nach dem Herausspalten der betreffenden Peptide noch über Disulfidbrücken verbunden bleiben (Abb. 2). Die wesentlichen Unterschiede sind: Das HMW-Kininogen kommt im

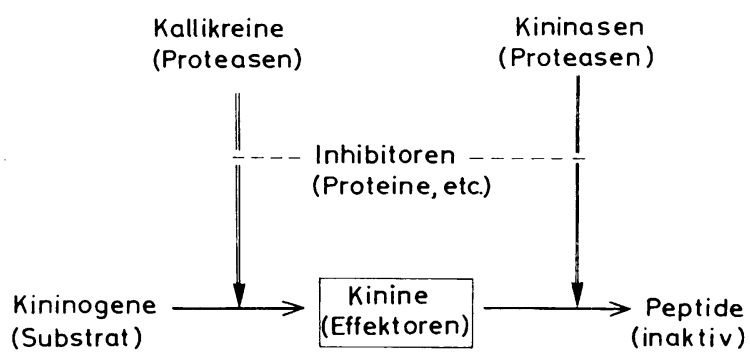

Abb. 1. Die einzelnen Komponenten des Kallikrein-Kinin-Systems und ihre Interaktionen (aus: Kallikrein in der Andrologie. Schirren, C. (ed.). Berlin: Grosse 1978 


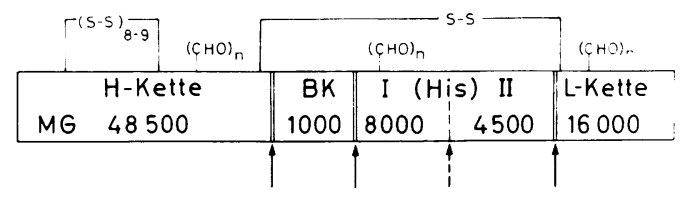

HMW-Kininogen Plasma-Kallikrein

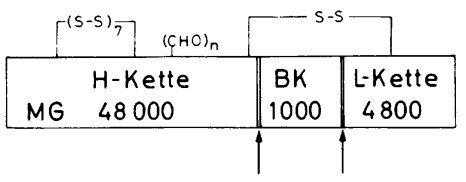

LMW-Kininogen Kininogenasen
Abb. 2. HMW- und LMW-Kininogen des Rindes. Schematische Darstellung des Aufbaus und der Spaltstellen für Organ- und PlasmaKallikreine. MG = Molekulargewicht, BK = Bradykinin, His = histidinreiche Peptide. $(\mathrm{CHO})_{\mathrm{n}}=$ Polysaccharidkette, $\mathrm{S}-\mathrm{S}=$ Disulfidbrücke (aus: Kallikrein in der Andrologie. Schirren, C. (ed.). Berlin: Grosse 1978

Plasma gebunden an Gerinnungsfaktoren, wie Faktor XI und Plasmapräkallikrein vor. Die Anlagerung erfolgt über die L-Kette und über das sehr basische histidinreiche Peptid. Das LMW-Kininogen besitzt diese Assoziationsfähigkeit nicht. Es enthält kein histidinreiches Peptid und seine L-Kette zeigt keine strukturelle Ähnlichkeit mit der L-Kette des HMW-Kininogens. Eine in vitro-Gerinnungsstörung, bedingt durch einen Mangel an HMW-Kininogen (Fitzgerald-Faktor) kann nicht durch LMW-Kininogen korrigiert werden.

Das Plasmakallikrein einerseits und die Drüsen-, Organ- oder Gewebskallikreine andererseits sind völlig verschiedene Enzyme. Der Unterschied besteht in ihren physikalisch-chemischen Eigenschaften, in ihrem Reaktionsverhalten gegenüber Substraten und Inhibitoren und in ihrem immunchemischen Verhalten. Die Organkallikreine ihrerseits (aus Pankreas, Speicheldrüsen und Harn) sind strukturell so eng miteinander verwandt, daß sie mittels immunchemischer und enzymatischer Methoden nach ihrem Herkunftsort nicht unterschieden werden können.

Das Kallikrein-Kinin-System des Plasmas und das der Organe dürfen nicht als in sich abgeschlossen betrachtet werden. Es gibt zahlreiche Verknüpfungspunkte mit anderen Systemen: Die Prostaglandinsynthese wird durch Kinine stimuliert, der cyclische GMPbzw. der cyclische AMP-Spiegel wird direkt bzw. indirekt über Prostaglandine erhöht. Mit dem Complementsystem besteht teils eine direkte, teils eine indirekte Verbindung und das Renin-Angiotensin-System ist ein direkter Gegenspieler des Kallikrein-Kinin-Systems.

Im Gegensatz zu den vielen Detailkenntnissen über die Enzymologie und Biochemie der Organkallikreine besitzen wir nur wenige gesicherte Erkenntnisse über ihre physiologische Wirkungsweise im Organismus. Aus den beobachtbaren Wirkungen, die nach exogener Zufuhr von Pankreaskallikrein vom Schwein - im Handel als Padutin auftreten, ist der Schluß naheliegend, daß auch endogene Organkallikreine ähnliche Wirkungen entfalten könnten: Das sind u. a. eine Stimulierung der intestinalen Resorption, eine Erhöhung der Spermienzahl und Spermienmotilität (Schill, 1975), eine Erhöhung der muskulären Glukoseaufnahme (Dietze u. Wicklmayr, 1977) und eine Steigerung der Zellproliferation (Rohen u. Peterhoff, 1975). Völlig offen ist dabei jedoch, wie das oral oder durch Injektion applizierte Gewebskallikrein zur Wirkung gelangt. Einige der anstehenden Fragen konnten mit Hilfe von Radioimmunoassays für Schweinepankreaskallikrein (Fink u. Güttel, 1978) und für menschliches Harnkallikrein (Mann u. Geiger, 1977) geklärt werden (Fink, 1979).

1. Es konnte gezeigt werden, daß auch Organkallikrein im Plasma vorkommt, was bislang als unwahrscheinlich galt, und zwar in einer mittleren Konzentration von 20 $\mathrm{ng} / \mathrm{ml}$. 
2. Bislang wurde allgemein angenommen, daß das im Harn ausgeschiedene Organkallikrein ausschließlich aus der Niere stammt bzw. dort synthetisiert wird. Fink konnte im Tierversuch zeigen, daß sowohl speziesfremdes als auch spezieseigenes, aber exogen zugeführtes Organkallikrein über die Niere ausgeschieden wird. Damit liegt der Schluß nahe, daß unter physiologischen Bedingungen auch endogenes, im Blut zirkulierendes Organkallikrein in den Urin ausgeschieden wird. Das von einer Reihe von Autoren vertretene Konzept der Identität von Nierenkallikrein und Harnkallikrein ist in dieser ausschließlichen Form demnach nicht länger aufrecht zu erhalten.

3. Weiter konnte im Tierversuch eindeutig gezeigt werden, daß Kallikrein aus dem Darm in immunologisch und enzymatisch aktiver Form resorbiert wird und sowohl im Plasma als auch in der Lymphe erscheint. Damit ist die Basis für die therapeutische Wirkung von oral appliziertem Kallikrein gegeben.

Dem Nieren- bzw. Harnkallikrein-Kinin-System schreibt man als direkten Gegenspieler des Renin-Angiotensin-Systems (die Kininase II ist identisch mit dem angiotensin converting encyme) eine spezielle Funktion in der Blutdruckregulation und im Elektrolythaushalt zu. Allgemein verbreitet ist die Annahme, das Kallikrein-Kinin-System wirke diuretisch und natriuretisch. In der Literatur findet man dazu aber ganz widersprüchliche Befunde (Ward u. Margolius, 1979). Man weiß bis heute noch nicht mit Sicherheit, was das Kallikrein-Kinin-System an der Niere tatsächlich bewirkt, ob es neben seiner Blutdruckregulation noch direkt an der Kontrolle der Elektrolyt- und Wasserausscheidung beteiligt ist oder nicht. Und wenn $\mathrm{ja}$, ob es einen natriuretischen oder antinatriuretischen Effekt hat.

Neuerdings gibt es Befunde, die speziell für die Beeinflußung des $\mathrm{Na}^{+} / \mathrm{K}^{+}$-Austausches am distalen Tubulus sprechen, z. B. hemmt Amilorid sowohl die biologische als auch die esterolytische Aktivität des Humanurinkallikreins (Margolius et al., 1979). Vielleicht sind wir einem generellen Wirkprinzip auf der Spur, das an allen Zellmembranen gilt.

Bei der essentiellen Hypertonie gibt es eine Untergruppe mit verminderter Harnkallikreinausscheidung. Bereits vor dem Manifestwerden einer Hochdruckerkrankung ist die verminderte Harnkallikreinausscheidung feststellbar. Die Regulationsfähigkeit im Elektrolythaushalt ist stark eingeschränkt (normalerweise reagiert z. B. der Organismus auf eine kochsalzarme Diät mit einer Erhöhung der Harnkallikreinausscheidung und vice versa). Die Arbeitsgruppe Overlack hat eine Studie mit essentiellen Hypertonikern durchgeführt, wobei Padutin als Langzeittherapie oral verabreicht wurde (Overlack et al., 1979). Nach einer Anlaufzeit von 2 Wochen begann sich der Blutdruck zu normalisieren. Auch die Harnkallikreinausscheidung normalisierte sich.

Höchst interessant war die Feststellung, die wir in Zusammenarbeit mit der obengenannten Arbeitsgruppe machten, daß es sich bei dem während der Therapie vermehrt ausgeschiedenen Harnkallikrein nicht um das applizierte Schweinepankreaskallikrein, sondern um endogenes Humanorgankallikrein handelte. Die Wirkung beruht offensichtlich nicht auf einem einfachen Substitutionseffekt, sondern darauf, daß eine Korrektur der gestörten Homöostase des K-K-Systems in dem Sinne erfolgt, daß ein defekter Syntheseoder Aktivierungsschritt "repariert“ wird - sei er nun genetisch bedingt oder erworben.

In diesem Zusammenhang ist erwähnenswert, daß das Bartters-Syndrom mit einer hohen Harnkallikreinausscheidung einhergeht. Bei der biochemischen Charakterisierung stellte man vor kurzem ein völlig abnormales Enzym fest, dessen biologische Aktivität stark vermindert war (Tamura et al., 1979). Ob diese Synthesestörung primär die Ursache ist für das Krankheitsbild oder ob die Störung sekundär auftritt, ob sie generalisiert oder an der Niere lokalisiert ist, bleibt noch dahingestellt. 
Es gibt viele Möglichkeiten für eine Störung der Homöostase des Kallikrein-Kinin-Systems. Nur die gleichzeitige Erfassung all seiner Komponenten und das gleichzeitige Messen der unmittelbar mitbetroffenen, veränderlichen Parameter werden uns die Klärung der vielen noch offenen Fragen näherbringen sowie eventuell neue Therapiemöglichkeiten eröffnen.

\section{Literatur}

Dietze, G., Wicklmayr, M.: Effekt von Bradykinin auf die Glukoseaufnahme durch die Muskulatur beim Menschen. Klin. Wochenschr. 55, 357-358 (1977). - Erdös, E. G.: The kinins - a status report. Biochem. Pharmacol. 25, 1563-1569 (1976). - Fink, E., Güttel, C.: Development of a radioimmunoassay for pig pancreatic kallikrein. J. Clin. Chem. Clin. Biochem. 16, 381-385 (1978). - Fink, E.: Habilitationsschrift. Medizinische Fakultät der Universität München, 1979. - Kato, H., Han, Y. N., Iwanaga, S., Hashimoto, N., Sugo, T., Fujii, S., Suzuki, T.: Mammalian plasma kininogens: Their structures and functions. In: Kininogenases-Kallikrein, Vol. 4. Haberland, G. L., Rohen, J. W., Suzuki, T. (eds.). Stuttgart: Schattauer 1977. - Mann, K., Geiger, R.: Radioimmunoassay of human urinary kallikrein. In: Kininogenases-Kallikrein, Vol. 4. Haberland, G. L., Rohen, J. W., Suzuki, T. (eds.), pp. 55-61. Stuttgart: Schattauer 1977. Margolius, H. S., Chao, J., Perlman, B., Westbury, M.: Identification of amphibian kallikrein and studies of kallikrein inhibition by amiloride. In: Proceedings Int. Symp. on Kinins, Tokyo, 1978. New York: Plenum Press 1979. - Overlack, A., Stumpe, K. O., Zywzock, W., Ressel, C., Krück, F.: Defect in kallikrein-kininsystem in essential hypertension and reduction of blood pressure by orally given kallikrein. In: Proceedings Int. Symp. on Kinins, Tokyo, 1978. New York: Plenum Press 1979. - Pisano, J. J., Austen, K. F.: Chemistry and biology of the kallikrein-kinin-system in health and disease. DHEW Publication No. (NIH) 76-791. Washington, D.C.: U.S. Government Printing Office 20-402. 1976. - Rohen, J. W., Peterhoff, J.: Stimulation of mitotic activity by kallikrein in the gastro intestinal tract of rats. In: Kininogenases-Kallikrein, Vol. 1. Haberland, G. L., Rohen, J. W. (eds.), pp. 57-66. Stuttgart: Schattauer 1975. - Schill, W.-B.: Influence of the kallikrein-kinin-system on human sperm motility in vitro. In: Kininogenases-Kallikrein, Vol. 2. Haberland, G. L., Rohen, J. W., Schirren, C., Huber, P. (eds.), pp. 47-57. Stuttgart: Schattauer 1975. - Sucuteri, E., Back, N., Haberland, G. L.: Kinins-pharmacodynamics and biological roles. Adv. Exp. Med. Biol. 70 (1976). - Tamura, Y., Matsuda, Y., Yamada, K., Nishikawa, T., Watanabe, M., Mikami, K., Takagi, T., Moriya, H., Kumagai, A.: Some aspects of urinary kallikrein in a patient with Bartter's syndrome. In: Proceedings Int. Symp. on Kinins, Tokyo, 1978. New York: Plenum Press 1979. Ward, P. E., Margolius, H. S.: Renal and urinary kallikreins. In the supplement to Vol. XXV. Handbook of experimental pharmacology. Bradykinin, Kallidin and Kallikrein. Erdös, E. G. (ed.). Heidelberg: Springer 1979.

\section{Kininsystem und Regulation des Muskelstoffwechsels}

Dietze, G., Wicklmayr, M., Günther, B., Lichtneckert, E., Böttger, I., Geiger, R., Waczek, S. L., Janetschek, P., Mehnert, H., Czempiel, H., Henftling, H. G., Fritz, H., Pabst, H. P., Heberer, G. (III. Med. Abt., Stoffwechsel und Endokrinologie, und Nuklearmed. Abt. des Akademischen Lehrkrankenhauses München-Schwabing, Nuklearmed. Abt. der TU und Chirurg. Klinik der Univ. München)

\section{Referat}

1925 bringt Frey das Estaunliche zuwege, als Chirurg eine biochemische Substanz zu entdecken [1]. Es handelt sich dabei um das Enzym Kallikrein, das aus Kininogen, einem großen Protein mit einem Molekulargewicht von ca. 40 000, die relativ kleinen Kinine mit einem Molekulargewicht von ca. 1000 zu spalten vermag. Diese Wirkungsweise des Kallikreins wurde erst 12 Jahre später von Werle aufgeklärt, als er die Freisetzung des Kinins Kallidin entdeckte [2]. Erst weitere 12 Jahre später gelang es dann Rocha e Silva un- 